\title{
Chandra Imaging of Gamma-Ray Binaries
}

\author{
O. Kargaltsev ${ }^{1, \star}$, B. Rangelov ${ }^{1}$, J. Hare ${ }^{1}$, and G.G. Pavlov ${ }^{2}$ \\ 1 George Washington University, Department of Physics, 725 21st St, NW, Washington, DC 20052 \\ 2 Pennsylvania State University, Department of Astronomy and Astrophysics, University Park, PA 16802
}

Received 24 Aug 2013, accepted 11 Nov 2013

Published online later

Key words X-rays: binaries stars: neutron pulsars: individual (B1259-63, LS 5039, LS I +61 303, HESS J0632+057, 1FGL J1018.6-5856)

\begin{abstract}
We review the multiwavelength properties of the few known gamma-ray binaries, focusing on extended emission recently resolved with Chandra. We discuss the implications of these findings for the nature of compact objects and for physical processes operating in these systems.
\end{abstract}

(c) 2006 WILEY-VCH Verlag GmbH \& Co. KGaA, Weinheim

\section{Introduction}

Thanks to recent advances in space-based X-ray, high energy (HE, GeV), and very high energy (VHE, TeV) $\gamma$-ray observations, an emerging population of high-mass gammaray binaries (HMGBs) has become an important topic in modern high-energy astrophysics. The five VHE HMGBs firmly detected in $\mathrm{TeV}$ and/or $\mathrm{GeV} \gamma$-rays, can be observationally separated into two types: (1) binaries where a rotation-powered pulsar interacts with the strong wind of the massive stellar companion (LS 2883/B1259-63), and (2) microquasars (LS 5039 and LS I +61 303). The types of the other two HMGB, HESS J0632+057 and HESS J1018589A/1FGL J1018.6-5856, are so far uncertain. The latter is the only HMGB coincident with the supernova remnant (G284.3-1.8). In addition to the five VHE HMGBs there are several other massive binary systems (e.g., Cygnus X-3, $\eta$ Carina) which were detected in the $\mathrm{GeV}$ band with Fermi LAT but so far lack the TeV detections. In most of the VHE binaries the $\mathrm{GeV}$ light curves are modulated with the orbital period determined from optical spectra and/or radio measurements. Below we summarize and compare the observational properties of the HMGBs focusing on imaging observations of VHE HGMBs with the Chandra $X$-ray Observatory $(C X O)$. We also discuss possible reasons for different multiwavelength (MW) manifestations of these systems.

\section{MW Properties of HMGBs}

All five VHE HMGBs are fairly bright in $\mathrm{GeV} \gamma$-rays and X-rays with the exception of HESS J0632+057 whose $\mathrm{GeV}$ emission (Hill et al. (2013)) has not been detected yet (the limit on its $\mathrm{GeV}$ luminosity is lower than the $\mathrm{GeV}$ luminosity of the other 4 VHE HMGBs). However,

* Corresponding author: e-mail: kargaltsev@gwu.edu
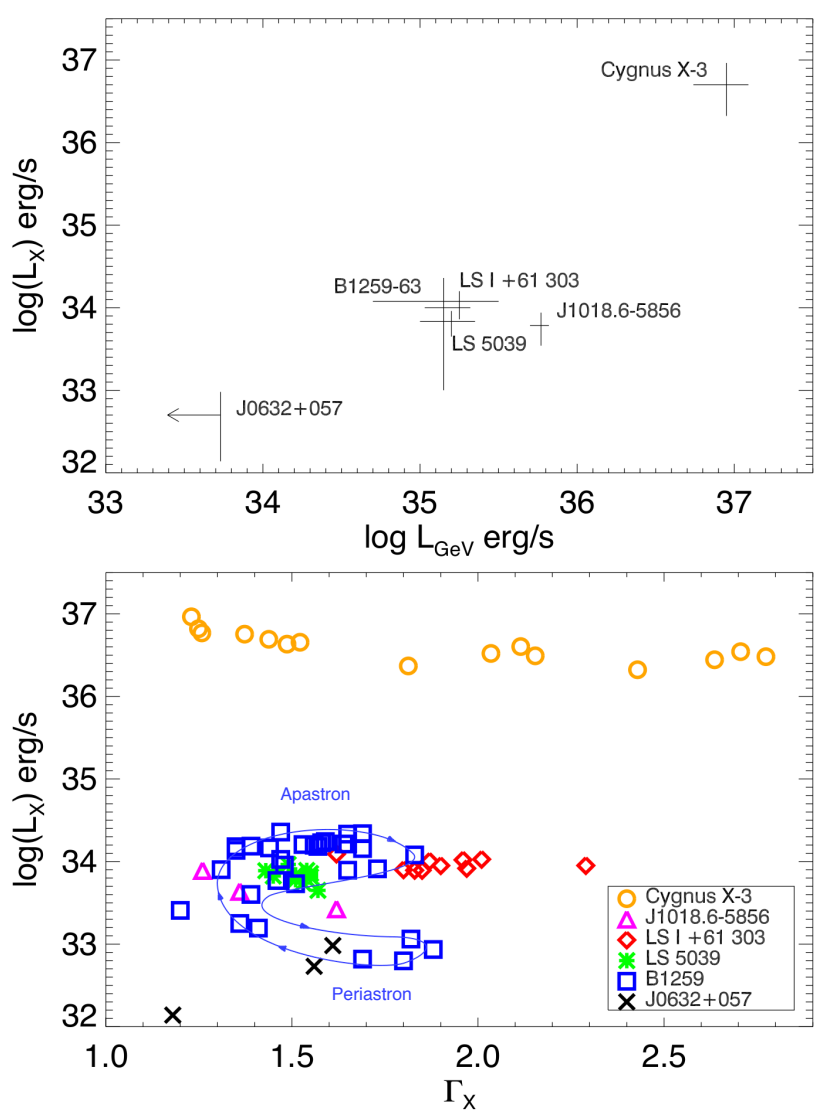

Fig. 1 X-ray luminosity $L_{X}$ vs. GeV luminosity $L_{G e V}$ (top), and $L_{X}$ vs. $\Gamma_{X}$ (bottom). The lines represent the observed parameter ranges as listed in Table 1 . The arrow on J0632+057 (top panel) shows the upper limit of its $\mathrm{GeV}$ luminosity. For Cygnus X-3 we only show a subset of the available data (Shrader et al.(2010)) for which satisfactory fits were obtained (excluding extremely hard or soft components). The blue curve with arrows (bottom) shows the evolution of B1259 parameters with the orbital phase. 

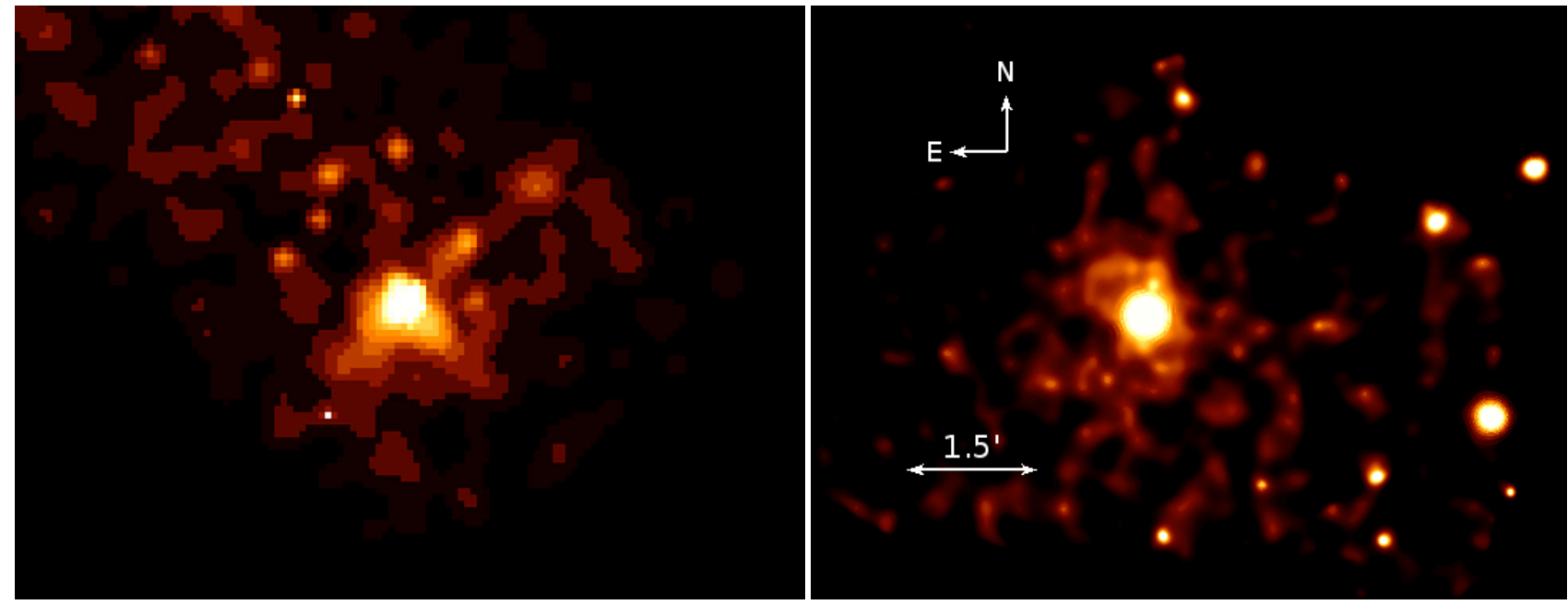

Fig. 2 Left: $28 \mathrm{ks}$ ACIS-I3 image ( $0.5-8 \mathrm{keV}$; binned to a pixel size of $1^{\prime \prime}$ and adaptively smoothed) showing extended emission surrounding LS 2883 (adopted from Pavlov et al. (2011)). Right: $38 \mathrm{ks}$ ACIS-I image (0.7 - $7 \mathrm{keV}$; binned to a pixel size of $1^{\prime \prime}$ and adaptively smoothed) of LS 5039 vicinity (see also Durant et al. (2011)).

given the somewhat lower $\mathrm{TeV}$ and $\mathrm{X}$-ray luminosities of HESS J0632+057, it is possible that the distance to this HMGB $(d=1.5 \mathrm{kpc}$; Aharonian et al. (2007)) is underestimated.

We compare the MW properties of the HMGBs presented in Table 1. While the small number of HMGBs prevents us from drawing any solid conclusions about this population of $\gamma$-ray emitters, we still notice some correlations between their MW properties (see also Dubus (2013) for more detailed discussion of MW properties). Fig. 1 shows the X-ray luminosity as a function of the GeV luminosity (top) and $\Gamma_{X}$ (bottom). Although the sample is small, one can notice from Fig. 1 that B1259, LS 5039, and LS I +61 303 inhabit the same locality on the plot, which suggests that they may be similar in their nature. We can also note that their $L_{\mathrm{TeV}}$ and $\Gamma_{\mathrm{TeV}}$ are similar to those of TeV PWNe (see Fig. 7 from Kargaltsev et al. (2013)). This would not be surprising if the source of power in these systems were a young pulsar, and the $\gamma$-ray radiation were due to ICS of the starlight. Unfortunately, with the exception of LS 2883/B1259-63, the nature of compact objects in the other four $\gamma$-ray binaries has not been confidently established yet.

The Fermi LAT spectra of the two microquasars, LS 5039 and LS I+61 303, and 1FGL J1018.6-5856 exhibit cut-offs in $2-6 \mathrm{GeV}$ range when modeled as powerlaws $(\Gamma=1.9-2.2)$ with exponential cut-offs. This is very similar to the $\mathrm{GeV}$ spectra of isolated pulsars. These systems show only modest variability levels in GeV compared to LS 2883 (Hadasch et al. (2012)). The more variable LS 2883, which is known to host the young pulsar B125963 , shows a noticeably harder spectrum $(\Gamma \approx 1.4)$ and substantially lower cut-off energy $(\approx 0.3 \mathrm{GeV})$ in the bright flaring state (Abdo et al. (2011)). Cygnus X-3 appears to exhibit a much softer $(\Gamma=2.5-2.7)$ spectrum when it is detected through its flares in $\mathrm{GeV}$ (Corbel et al. (2012)).

\section{Extended X-ray emission.}

The exquisite angular resolution of $C X O$ resolved extended emission from HMGBs for the first time (Fig. 2). We consider these findings secure for two HMGBs (LS 2883, LS 5039) while in other cases additional observations are needed to firmly establish the existence and the origin of the extended emission (see below). Since HESS J0632+057 has not been observed in the imaging mode with $C X O$ few constraints exist regarding the presence of extended emission. Based on similarities between HESS J0632+057 and LS 2883 (i.e., long period, large eccentricity, and hints of extended radio emission), one may expect to detect such emission around HESS J0632+057 as well, if the compact object is also a pulsar. The existing $C X O$ observations of all HMGBs are summarized in Table 2. Radio observations of HMGBs also revealed extended (and sometimes variable) structures (Moldón et al. (2013)) which however have much smaller scales $(\sim 3-500 \mathrm{AU})$ than the X-ray nebulae described below.

LS 2883/B1259-63: Extended emission around LS 2883/B1259-63 has been reported by Pavlov et al. (2011) from a 28 ks $C X O$ observation (see Fig. 2 left). It has been suggested that the emission could be a shocked pulsar wind escaping the binary near apastron. Since then two more $C X O$ observations have been carried out. The extended emission was detected with high confidence and was found to be variable both in morphology and flux. The detailed results of these two observations are discussed in our forthcoming paper.

LS 5039: Durant et al. (2011) analyzed the $38 \mathrm{ks} C X O$ ACIS observation and found evidence of nonthermal emission extending up to $1^{\prime}$ from LS 5039 (see Fig. 2, right). The spectrum could be fitted by an absorbed power-law (PL) model with $\Gamma=1.9 \pm 0.3$ and unabsorbed $0.5-8 \mathrm{keV}$ luminosity $L_{X} \simeq 1 \times 10^{32} \mathrm{erg} \mathrm{s}^{-1}$ for a plausible distance of $2.5 \mathrm{kpc}$. 

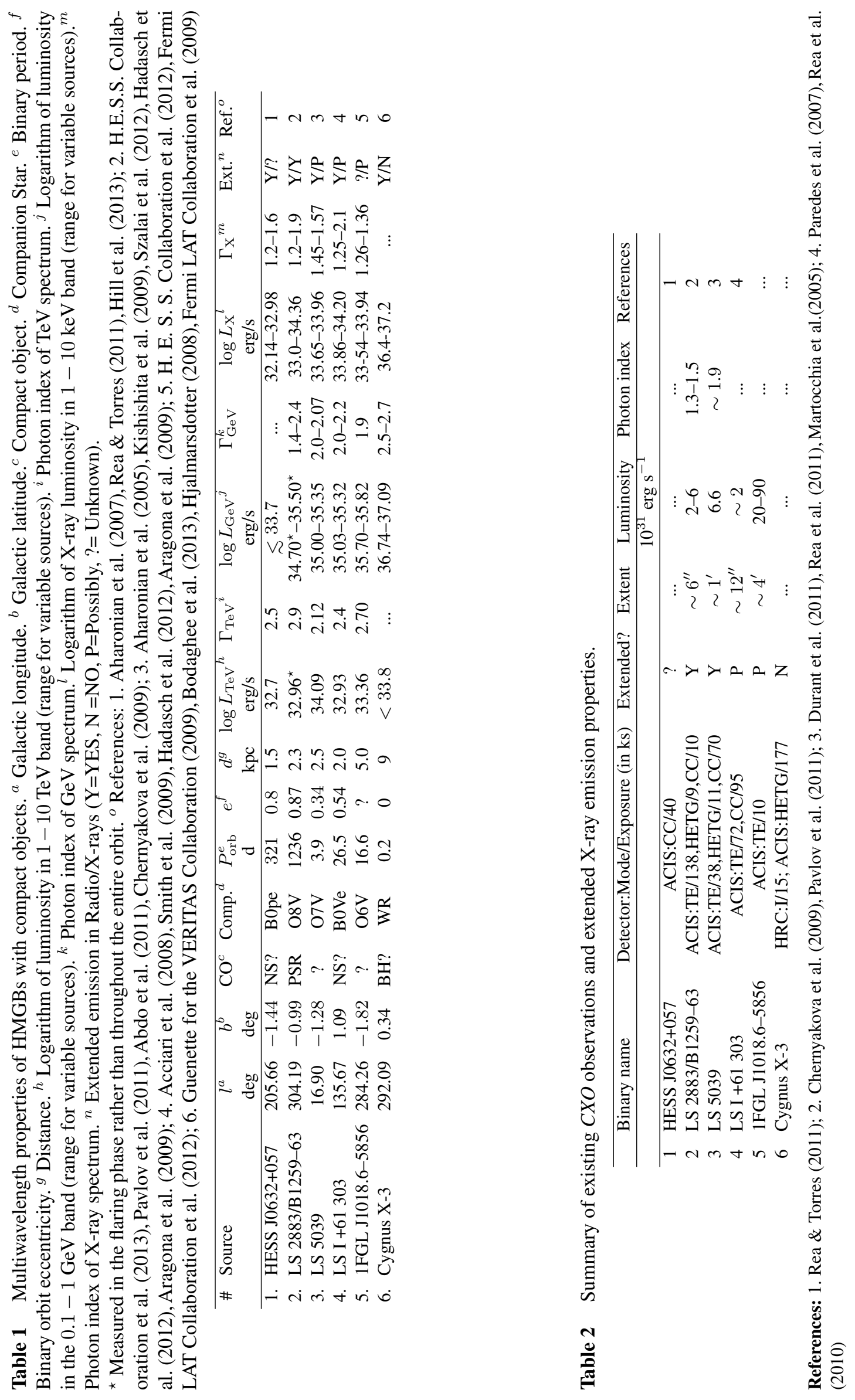
The non-isotropic morphology and the hard spectrum of the observed emission argue against the dust halo (which was also detected in this observation on a smaller angular scale). The presence of the nonthermal nebula suggests that the compact object is a pulsar. If so, the termination shock in the pulsar wind would probably occur at much smaller distances than those typical for isolated pulsars with PWNe, and, since the binary orbit is rather tight, the pulsar wind should interact with the denser O-star wind (compared to LS 2883). Such interaction and the accompanying mixing due to the instabilities would probably prevent the formation of any fast bulk flow (Bosch-Ramon \& Barkov (2011)), however, some ultra-relativistic electrons can still escape via diffusion to large distances before their energies become too low to emit synchrotron X-rays.

1FGL J1018.6-5856: The donor star in this binary is similar to that of LS 5039, but the orbital period is a factor of 4 longer, and the distance is likely a factor of two larger. Similar to LS 5039, arcminute-scale extended emission is seen around the binary (see Fig. 3) in the $10 \mathrm{ks} C X O$ observation (Pavlov et al. (2011)). The observed spectrum consists of two components, the softer of which is likely the SNR emission. The shell of G284.3-1.8 is clearly seen to the north of the binary (see Fig. 4), and the X-ray emission adjacent to the shell in the north has a soft X-ray spectrum compatible with the softer component of the spectrum extracted from the vicinity of 1FGL J1018.6-5856. The origin of the harder X-ray component is so far unclear. Compared to LS 5039 , the angular extent of the hard emission is a factor of 4-5 larger (at a factor of 2 larger distance) while the spectrum appears to be much harder than that of the extended emission around LS 5039 (see Fig. 3). The forthcoming 80 ks $C X O$ observation will shed more light on this interesting source.

LS I +61 303: The extended emission has been reported by Paredes et al. (2007) based on a $50 \mathrm{ks} C X O$ observation carried out with the ACIS-I array (Paredes et al. (2007)). It was interpreted as possibly being thermal Bremsstrahlung created from a large-scale outflow from LS I +61 303 which interacts with the nearby hot and dense ISM. Another possible explanation is that this is non-thermal synchrotron or inverse Compton emission produced by particles beyond the termination region of the pulsar wind (Paredes et al. (2007)).The presence of the extended emission has been disputed by Rea et al. (2010) who argued that there was no significant extension observed from this source based on the ACIS continuous clocking (CC) mode data, which, however, only offers one-dimensional imaging capability and suffers from a much larger background compared to the conventional imaging.

Cygnus X-3: Cyg X-3 does not show extended emission in the $C X O$ images which indicates a lack (or reduced numbers) of ultra-relativistic electrons. Cyg X-3 shows orbital modulation in both soft and hard states that is stronger in softer states. The modulation amplitude decreases with increasing energy in the hard, intermediate, and very high states (Weng et al. (2013)). The sporadic GeV emission reported by Bodaghee et al. (2013), and Corbel et al. (2012), is likely to be associated with the state transitions although some orbital phases has also been seen (Fermi LAT Collaboration et al. (2009)). The existence of extended radio jets, which qualifies Cyg X-3 as a microquasar, is common for both $\mathrm{BH}$ and NS X-ray binaries (the former is favored for Cyg X-3 when both radio and X-ray emission are considered (Zdziarski et al. (2010)). However, the presence of the jets (the defining feature of microquasars), often associated with the particle acceleration sites, does not appear to guarantee copious $\mathrm{GeV}$ or $\mathrm{TeV} \gamma$-ray production neither for $\mathrm{BH}$ nor for NS HMXBs.

Finally, the most populous group of HMXBs which are not microquasars are the accreting $\mathrm{X}$-ray pulsars (such as the wind-fed Vela X-1 or $1 \mathrm{~A} 0535+262$ ) which do not show nonthermal components extended to $\gamma$-rays. X-ray emission from these systems is dominated by thermal emission with Comptonized hard X-ray tail and powered by either accretion disk or hot accreting column above the surface. The apparent deficit of ultra-relativistic electrons in these systems suggests that they are inefficient accelerators.

Acknowledgements. This work was partly supported by Chandra award GO2-13085 and by NASA grant NNX09AC81G.

\section{References}

Abdo, A. A., Ackermann, M., Ajello, M., et al. 2011, ApJ , 736, L11

Acciari, V. A., Beilicke, M., Blaylock, G., et al. 2008, ApJ , 679, 1427

Acciari, V. A., Aliu, E., Araya, M., et al. 2011, ApJ , 733, 96

Aharonian, F., Akhperjanian, A. G., Aye, K.-M., et al. 2005, Science, 309, 746

Aharonian, F. A., Akhperjanian, A. G., Bazer-Bachi, A. R., et al. 2007, AAP, 469, L1

Aragona, C., McSwain, M. V., Grundstrom, E. D., et al. 2009, ApJ , 698, 514

Bodaghee, A., Tomsick, J. A., Pottschmidt, K., et al. 2013, arXiv:1307.3264

Bosch-Ramon, V., \& Barkov, M. V. 2011, AAP, 535, A20

Caballero, I. 2009, Ph.D. Thesis

Chernyakova, M., Neronov, A., Aharonian, F., Uchiyama, Y., \& Takahashi, T. 2009, MNRAS , 397, 2123

Corbel, S., Dubus, G., Tomsick, J. A., et al. 2012, MNRAS , 421, 2947

Dubus, G. 2013, arXiv:1307.7083

Durant, M., Kargaltsev, O., Pavlov, G. G., Chang, C., \& Garmire, G. P. 2011, ApJ , 735, 58

Fermi LAT Collaboration, Abdo, A. A., Ackermann, M., et al. 2009, Science, 326, 1512

Fermi LAT Collaboration, Ackermann, M., Ajello, M., et al. 2012, Science, 335, 189

Guenette, R., \& for the VERITAS Collaboration 2009, arXiv:0908.0714

Hadasch, D., Torres, D. F., Tanaka, T., et al. 2012, ApJ , 749, 54

H. E. S. S. Collaboration, Abramowski, A., Acero, F., et al. 2012, $A A P, 541$, A5

H.E.S.S. Collaboration, Abramowski, A., Acero, F., et al. 2013, $A A P, 551$, A94 

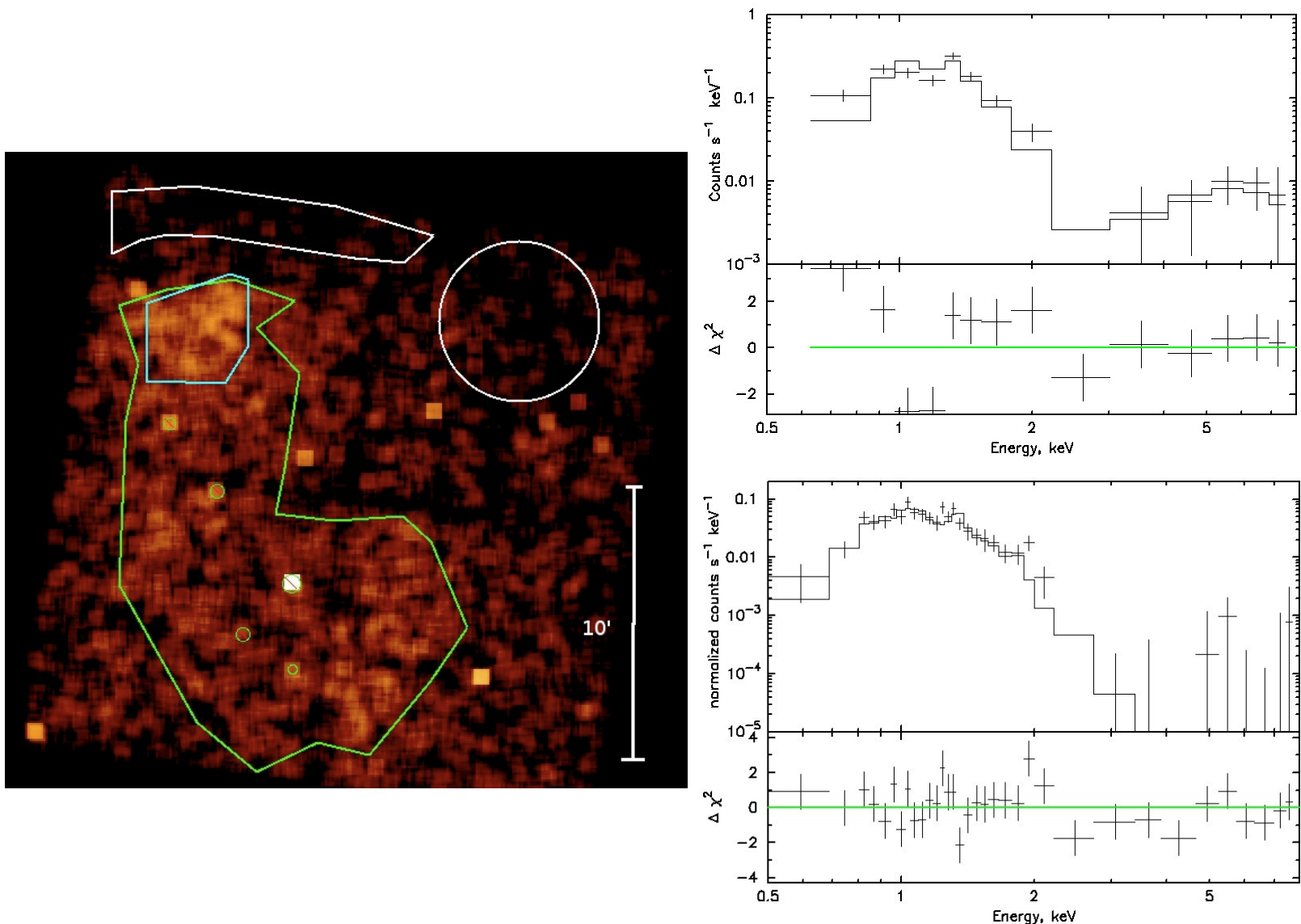

Fig. 3 Left: ACIS-I $10 \mathrm{ks}$ image (0.5-8 keV) of the field of 1FGL J1018-5856. Regions for preliminary spectral analysis of the apparent extended emission are shown: the green line encompasses the entire diffuse emission, and the blue line only the soft northern lobe. Appropriate background regions are marked in white. Point sources were excluded from the extraction region, e.g., J1018 (the brightest source near the centre). Right: Spectral fit to the whole nebula (MEKAL+PL model, top) and the soft northern lobe (MEKAL, bottom). The MEKAL temperatures are similar for both regions ( $0.22 \mathrm{keV}$ and $0.23 \mathrm{keV}$, respectively), but only the whole nebula spectrum requires a hard component. Although significant, its photon index is very poorly defined.
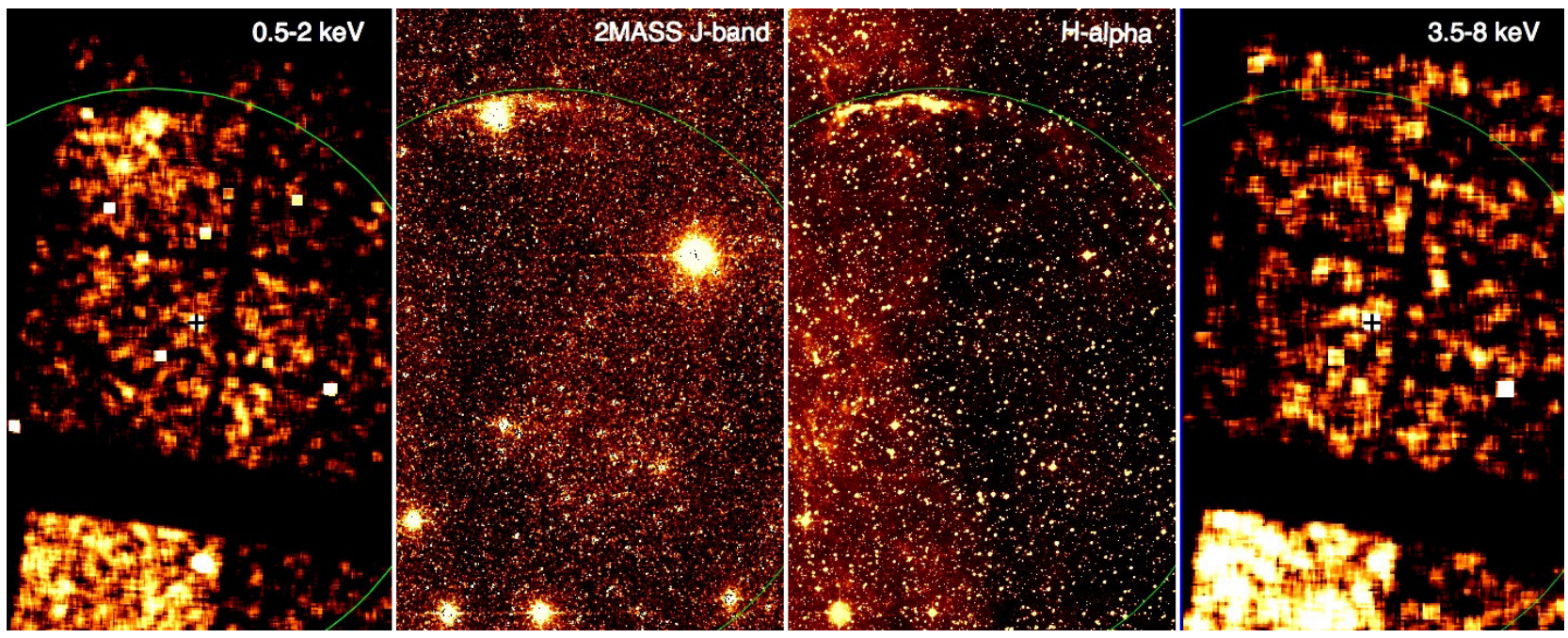

Fig. 4 Field in the vicinity of J1018 in two X-ray bands, with auxiliary NIR-optical data. Of particular interest is the filament seen in the two central panels, which appears to be a section of a circle and located just beyond the extent of the X-ray emission. 
Hill, A. B., Caliandro, A., \& on behalf of the Fermi-LAT collaboration 2013, arXiv:1304.2308

Hjalmarsdotter, L. 2008, American Institute of Physics Conference Series, 1054, 13

Kargaltsev, O., Rangelov, B., \& Pavlov, G. G. 2013, arXiv:1305.2552

Kendziorra, E., Kretschmar, P., Pan, H. C., et al. 1994, AAP, 291, L31

Kishishita, T., Tanaka, T., Uchiyama, Y., \& Takahashi, T. 2009, ApJ , 697, L1

Martocchia, A., Motch, C., \& Negueruela, I. 2005, AAP, 430, 245

Moldón, J., Ribó, M., \& Paredes, J. M. 2013, arXiv:1306.2830

Paredes, J. M., Ribó, M., Bosch-Ramon, V., et al. 2007, ApJ , 664, L39

Pavlov, G. G., Chang, C., \& Kargaltsev, O. 2011, ApJ , 730, 2

Pavlov, G. G., Misanovic, Z., Kargaltsev, O., \& Garmire, G. P. 2011, The Astronomer's Telegram, 3228, 1

Rea, N., Torres, D. F., van der Klis, M., et al. 2010, MNRAS , 405, 2206

Rea, N., Torres, D. F., Caliandro, G. A., et al. 2011, MNRAS , 416, 1514

Rea, N., \& Torres, D. F. 2011, ApJ , 737, L12

Shrader, C. R., Titarchuk, L., \& Shaposhnikov, N. 2010, ApJ , 718, 488

Smith, A., Kaaret, P., Holder, J., et al. 2009, ApJ , 693, 1621

Szalai, T., Sarty, G. E., Kiss, L. L., et al. 2012, IAU Symposium, 282,331

Weng, S.-S., Zhang, S.-N., Ge, M.-Y., Li, J., \& Zhang, S. 2013, ApJ , 763, 34

Zdziarski, A. A., Misra, R., \& Gierliński, M. 2010, MNRAS , 402, 767 